\title{
User guides for biologists to learn computational methods
}

\section{Dokyun $\mathrm{Na}^{*}$ \\ Department of Biomedical Engineering, Chung-Ang University, Seoul 06974, Republic of Korea \\ (Received Jan 17, 2020 / Revised Feb 10, 2020 / Accepted Feb 11, 2020)}

System-wide studies of a given molecular type are referred to as "omics." These include genomics, proteomics, and metabolomics, among others. Recent biotechnological advances allow for high-throughput measurement of cellular components, and thus it becomes possible to take a snapshot of all molecules inside cells, a form of omics study. Advances in computational modeling methods also make it possible to predict cellular mechanisms from the snapshots. These technologies have opened an era of computation-based biology. Component snapshots allow the discovery of gene-phenotype relationships in diseases, microorganisms in the human body, etc. Computational models allow us to predict new outcomes, which are useful in strain design in metabolic engineering and drug discovery from protein-ligand interactions. However, as the quantity of data increases or the model becomes complicated, the process becomes less accessible to biologists. In this special issue, six protocol articles are presented as user guides in the field of computational biology.

Keywords: computational biology, machine learning, microbiome, Ribo-seq, drug discovery

\section{Introduction}

Computational methods to gain insight into biological systems, including the evolution of genes and proteins, were first introduced in the 1960s (Ouzounis and Valencia, 2003). Since then, many computational methods have been developed to analyze protein/nucleotide sequences and to predict protein structures. In the era of omics, molecules inside cells (nucleotide sequences, transcripts, proteins) can be captured as a snapshot by high-throughput technologies. Based on the snapshots, computational models can be developed to fill the gaps between snapshots and to predict new outcomes.

Rapid advances in fields associated with computational bio-

${ }^{*}$ For correspondence. E-mail: blisszen@cau.ac.kr; Tel.: +82-2-820-5690; Fax: +82-2-814-2651

Copyright (c) 2020, The Microbiological Society of Korea logy, such as bioinformatics and systems biology, have allowed deeper insight into the mechanisms of cells, the relationship between cell components and diseases, etc. As the methods have become more sophisticated, they also have become less accessible to biologists. This special issue presents six different articles describing protocols that can be used readily by biologists and bioengineers. The aim of this issue is to introduce step-by-step instructions for scientists who are not familiar with computational approaches.

\section{Microbiome analysis}

The human body is colonized by at least 10 times more microorganisms than human cells that comprise it (NIH Human Microbiome Project [HMP] Working Group et al., 2009). This community of microorganisms (known as a microbiome) has been an attractive source of new information in understanding human diseases and the physiology of environmental microbes (Gilbert et al., 2018). Clear differences between the influence of organisms in the microbiome and the influence of genetic variation can be shown in cases such as obesity, autism, and allergies (Gilbert et al., 2018). Recent research has led to novel treatments such as, for example, fecal translation in the case of Clostridium difficile infection.

Traditional approaches to analyze microorganism communities include isolating bacterial species by cultivating them on media under controlled conditions. This approach is not always successful in quantifying microorganisms and may overlook many species because of differences in media requirements between species. Recently, next-generation sequencing (NGS) of $16 \mathrm{~S}$ rRNA has allowed analysis of microorganism communities in the human body or the surrounding environment. To analyze the $16 \mathrm{~S}$ rRNA sequences, they must be pre-processed, mapped to known microorganism species, and analyzed taxonomically, all of which can be achieved using computer systems. Three articles are included in this special issue: microbiome analysis from 16S rRNA sequences (Kim et al., 2020), analysis of environmental microbiomes (Jo et al., 2020), and machine-learning methods for microbiome analysis (Namkung, 2020). These guides will not only assist in analysis, but also expand understanding of microbiomes.

Jo et al. (2020) introduce a step-by-step protocol for the analysis of a microbial community obtained from shotgun metagenomic sequencing by using in silico tools. The community may be sampled from soil, deep water, the human body, or other microbiomes of interest. The article includes 
pre-processing of metagenomic datasets, taxonomy profiling, and functional annotation of sequenced genes to infer the function and physiology of the community.

Kim et al. (2020) introduce a protocol for NGS-based microbiome studies by analyzing the $16 \mathrm{~S}$ rRNA sequences of a microbial community. The article includes pre-process sequencing of raw data, diversity analysis, and taxonomic classification. This protocol article uses a QIIME2 core installed on a virtual machine, which is relatively easy to follow for those who are not bioinformaticians.

Namkung (2020) introduces fundamental knowledge on machine-learning algorithms and clustering/dimensionality reduction methods for the development of risk-prediction models and for analysis of microbiome profiles, respectively. Python is used to build a code to cluster microbiome profiles or to predict risk probability from the profiles. Specifically, the sklearn library is used for clustering, principle component analysis, and support vector machine.

\section{Ribo-Seq analysis}

NGS has influenced methods of conducting experiments and understanding underlying cellular mechanisms. NGS has been utilized to discover genomic sequences, to analyze the relationship between genetic variation and disease phenotype, to reconstruct metabolic pathways displaying physiological behaviors, to identify microorganisms living in the gut, etc.

In general, RNA-seq has been used widely to measure the abundance of transcripts in a cell (known as a transcriptome) (Nagalakshmi et al., 2008). A transcriptome provides more information than genomic data because transcript abundance is correlated with the activity of working genes. With a transcriptome, we can find which genes are working actively and determine how active they are. Despite this usefulness, transcript abundance may not be correlated directly with protein levels. Proteins perform a wide range of functions and define the status of cells. Therefore, in order to understand cellular systems and behaviors accurately, the entire complement of proteins in a cell (known as a proteome) should be measured. At present, it is still difficult to measure a whole proteome.

Ribo-seq has been developed to measure the abundance of transcripts that are translated actively (known as a translatome), which is correlated directly with protein levels, by footprinting ribosome-bound transcripts (Ingolia et al., 2009). In the early days, Ribo-Seq was used to study translation initiation, codon adaptation, translation elongation, translational control, etc. These days, Ribo-Seq is used to assess the levels of proteins.

Choe et al. (2020) introduce a step-by-step guide for RiboSeq analysis for those who are not bioinformaticians using a Simple Translatome Analysis Tool for Ribo-Seq (STATR). The article discusses how to conduct Ribo-Seq experiments and process raw data, as well as genome-wide differential expression analysis. The authors analyze the actively transcribing genes of the multi-drug resistance pathogen, Staphylococcus aureus, and find that the accessory gene regulator (Agr), a major regulator of virulence and pathogenesis, is translated in response to linezolid.

\section{Metabolic engineering}

Engineering microorganisms to construct microbial cell factories has gained attention as a sustainable technology alternative to current chemical factories. According to recent reports, refinery chemicals, energies and fuels, plastics, cosmetics, and even drugs are produced from microorganisms (Lee et al., 2012). However, metabolic networks and their genetic regulatory systems are wired in a highly complicated way and therefore it is difficult to intuitively predict the outcomes of genetic engineering of microorganisms. To understand cellular networks, omics data have been measured (genome, transcriptome, metabolome, proteome, etc.) and analyzed to depict a big picture of living organisms. Recently, computational methods have been developed to predict the outcome of gene modifications (Kabimoldayev et al., 2018; $\mathrm{Gu}$ et al., 2019).

Jeon et al. (2020) introduce a method to simulate a specific growth rate and theoretical production yield of succinic acid as an example by using COBRApy. Simulation and prediction of metabolic systems in microorganisms would stimulate the construction of microbial cell factories efficiently.

\section{Drug discovery}

Classical drug discovery strategy for a single drug requires 10-20 years to develop and the process costs approximately $\$ 1.8$ billion (Paul et al., 2010). In the drug discovery process, hit discovery, lead optimization, and preclinical tests require approximately 5-10 years (Marx et al., 2016). In preclinical tests, many drug candidates are withdrawn due to unexpected cardio- or hepato-toxicity. Fortunately, recent rapid advances in computer science allow for the development of prediction models for virtual screening. In addition, computational models of protein-drug interactions and machinelearning models for absorption, distribution, metabolism, excretion, and toxicity (ADME-Tox) have been developed using deep learning algorithms.

Shaker et al. (2020) introduce a user guide for in silico drug discovery that includes protein structure prediction, active site prediction, protein-drug interaction (docking), and ADMETox prediction, a virtual strategy for drug discovery. This might encourage biologists, even those who are not familiar with computer science, to carry out research on drug discovery.

\section{Acknowledgments}

This work was supported by National Research Foundation of Korea (NRF) grants funded by the Korea government (MSIT) (No. NRF-2018R1A5A1025077 and NRF-2019M3E5D4065682).

\section{References}

Choe, D., Palsson, B., and Cho, B.K. 2020. STATR: A simple analysis pipeline of Ribo-Seq in bacteria. J. Microbiol. 58, 217-226.

Gilbert, J.A., Blaser, M.J., Caporaso, J.G., Jansson, J.K., Lynch, S.V., and Knight, R. 2018. Current understanding of the human microbiome. Nat. Med. 24, 392-400. 
Gu, C., Kim, G.B., Kim, W.J., Kim, H.U., and Lee, S.Y. 2019. Current status and applications of genome-scale metabolic models. Genome Biol. 20, 121.

Ingolia, N.T., Ghaemmaghami, S., Newman, J.R., and Weissman, J.S. 2009. Genome-wide analysis in vivo of translation with nucleotide resolution using ribosome profiling. Science 324, 218 223.

Jeon, J. and Kim, H.U. 2020. Setup of a scientific computing environment for computational biology: Simulation of a genomescale metabolic model of Escherichia coli as an example. J. Microbiol. 58, 227-234.

Jo, J., Oh, J., and Park, C. 2020. Microbial community analysis using high-throughput sequencing technology: a beginner's guide for microbiologists. J. Microbiol. 58, 176-192.

Kabimoldayev, I., Nguyen, A.D., Yang, L., Park, S., Lee, E.Y., and Kim, D. 2018. Basics of genome-scale metabolic modeling and applications on C1-utilization. FEMS Microbiol. Lett. 365, fny241.

Kim, H., Kim, S., and Jung, S. 2020. Instruction of microbiome taxonomic profiling based on $16 \mathrm{~S}$ rRNA sequencing. J. Microbiol. 58, 193-205.

Lee, J.W., Na, D., Park, J.M., Lee, J., Choi, S., and Lee, S.Y. 2012. Systems metabolic engineering of microorganisms for natural and non-natural chemicals. Nat. Chem. Biol. 8, 536-546.

Marx, U., Andersson, T.B., Bahinski, A., Beilmann, M., Beken, S.,
Cassee, F.R., Cirit, M., Daneshian, M., Fitzpatrick, S., Frey, O., et al. 2016. Biology-inspired microphysiological system approaches to solve the prediction dilemma of substance testing. ALTEX 33, 272-321.

Nagalakshmi, U., Wang, Z., Waern, K., Shou, C., Raha, D., Gerstein, M., and Snyder, M. 2008. The transcriptional landscape of the yeast genome defined by RNA sequencing. Science 320, 13441349.

Namkung, J. 2020. Machine learning methods for microbiome studies. J. Microbiol. 58, 206-216.

NIH HMP Working Group, Peterson, J., Garges, S., Giovanni, M., McInnes, P., Wang, L., Schloss, J.A., Bonazzi, V., McEwen, J.E., Wetterstrand, K.A., et al. 2009. The NIH human microbiome project. Genome Res. 19, 2317-2323.

Ouzounis, C. and Valencia, A. 2003. Early bioinformatics: The birth of a discipline - a personal view. Bioinformatics 19, 2176-2190.

Paul, S.M., Mytelka, D.S., Dunwiddie, C.T., Persinger, C.C., Munos, B.H., Lindborg, S.R., and Schacht, A.L. 2010. How to improve R\&D productivity: The pharmaceutical industry's grand challenge. Nat. Rev. Drug Discov. 9, 203-214.

Shaker, B., Yu, M.S., Lee, J., Lee, Y., Jung, C., and Na, D. 2020. User guide for the discovery of potential drugs via protein structure prediction and ligand docking simulation. J. Microbiol. 58, 235-244. 